\title{
Off-Axis Dipole Forces in Optical Tweezers by an Optical Analog of the Magnus Effect
}

\author{
Robert J. C. Spreeuw ${ }^{*}$ \\ Van der Waals-Zeeman Institute, Institute of Physics, University of Amsterdam, P.O. Box 94485, 1090 GL Amsterdam, The Netherlands
}

(Received 15 May 2020; accepted 2 November 2020; published 1 December 2020)

\begin{abstract}
It is shown that a circular dipole can deflect the focused laser beam that induces it and will experience a corresponding transverse force. Quantitative expressions are derived for Gaussian and angular top hat beams, while the effects vanish in the plane wave limit. The phenomena are analogous to the Magnus effect, pushing a spinning ball onto a curved trajectory. The optical case originates in the coupling of spin and orbital angular momentum of the dipole and the light. In optical tweezers the force causes off-axis displacement of the trapping position of an atom by a spin-dependent amount up to $\lambda / 2 \pi$, set by the direction of a magnetic field. This suggests direct methods to demonstrate and explore these effects, for instance, to induce spin-dependent motion.
\end{abstract}

DOI: 10.1103/PhysRevLett.125.233201

A common practice in many branches of sports is to send a ball onto a curved trajectory by giving it a spin. In this famous example of the Magnus effect [1], the spinning ball deflects the stream of air around it and is pushed sideways by the reaction force perpendicular to its forward velocity. In analogy, we may ask if a rotating dipole in an atom may similarly deflect a beam of light and thereby be pushed by a force perpendicular to the light beam. Darwin already remarked that, for circular dipoles, "... the wave front of the emitted radiation faces not exactly away from the origin, but from a point about a wave-length away from it" [2]. A recent experiment confirmed that an atomic circular dipole can indeed appear to be displaced from its true location, due to the emitted spiral-shaped wave front [3]. Circular dipoles provide perhaps the simplest example of the intrinsic coupling of spin and orbital angular momentum in nonparaxial light fields [4-13]. Such fields, in the form of tightly focused laser beams, are of central importance in a rapidly growing range of experiments involving (arrays of) optical tweezers [14-24]. These are developed as precise tools to hold and manipulate single atoms or molecules at the quantum level, in creating platforms for quantum simulation and computation, as well as for quantum sensing and atomic clocks [25,26].

Here we predict that an atomic circular dipole can deflect the centered focused laser beam that induces it. Conversely, the atom will experience a transverse force when on axis [27]. An important consequence of this force can be seen in the off-axis displacement of the trapping potential created

Published by the American Physical Society under the terms of the Creative Commons Attribution 4.0 International license. Further distribution of this work must maintain attribution to the author(s) and the published article's title, journal citation, and DOI.

by an optical tweezer [24]. Thus, rather than "seeing an atom where it is not" $[3,29,30]$, here we describe its counterpart of "trapping an atom where the focus is not" [14,24,31]. A simple geometric argument based on light scattering shows that the true displacement of the trapping potential is in fact a direct consequence of the apparent

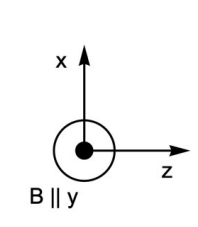

(a)

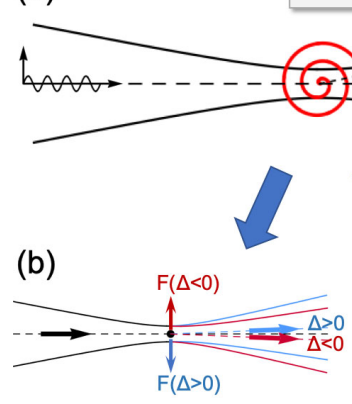

FIG. 1. Optical analog of the Magnus effect. (a) A linearly polarized $(\mathbf{E} \| x)$, focused laser induces a circular dipole ( $x z$ plane) on a $j=0 \rightarrow j^{\prime}=1\left(\Delta m_{j}=1\right)$ transition, with a magnetic field $\mathbf{B} \| y$ setting the quantization axis. The spiral wave scattered by the circular dipole interferes with the incident wave, producing two effects: (b) The incident beam is deflected in the $x z$ plane, with corresponding reaction force on the atom, transverse to the beam. The direction changes sign with the detuning from the atomic resonance. (c) In an optical tweezer ("red" detuning, $\Delta<0$ ), the transverse force shifts the trapping position away from the optical axis by an amount $\chi=\lambda / 2 \pi$. See Fig. 3 for a far off-resonance case. 
displacement of an emitting circular dipole $[2,3]$. Tweezer trap displacements have previously been calculated numerically, for specific beam shapes, in terms of vector and tensor light shifts [14,31]. The geometric argument given here directly shows that, for a circular dipole, the displacement is simply $\pm \lambda= \pm \lambda / 2 \pi$ and is remarkably independent of many parameters, including the laser detuning, trap frequency, and even the detailed shape of the trap. A comparison of a Gaussian beam with an angular top hat beam illustrates this. This profound insight provides the basis for state-dependent manipulation of atomic motion within the tweezer.

We describe these effects in terms of interference between the focused incident beam with the wave scattered by the circular dipole, see Fig. 1. In the optical theorem, such interference is used to describe the attenuation of light in terms of the forward scattering amplitude [32]. In contrast, here we concentrate on beam deflection, as a consequence of the tilt of the spiral wave front with respect to the incident wave. Two simple atomic-level schemes serve as examples; (i) a $j=0 \rightarrow j^{\prime}=1$ and (ii) a $j=1 \rightarrow$ $j^{\prime}=0$ transition. Case (i) is conceptually simpler and most suited to observe the Magnus-like deflection of a weak, near-resonant probe beam. Case (ii) offers interesting extra opportunities in the usual far off-resonance regime of optical tweezer experiments.

Starting with case (i), the $j=0 \rightarrow j^{\prime}=1$ transition, we focus a linearly polarized $(\mathbf{E} \| x)$, monochromatic laser onto a single atom placed in the origin, see Fig. 1. A magnetic field $\mathbf{B} \| y$ defines the quantization axis and splits the excited state into three $\left|j, m_{j}\right\rangle_{y}$ sublevels, separated by the Zeeman shift $\sim \mu_{B} B / \hbar$, with $\mu_{B}$ the Bohr magneton [33]. We tune the laser close to the $\Delta m_{j}=+1$ transition, with a detuning $\Delta=\omega_{L}-\omega_{0}$ small compared to the Zeeman shift, so that the $\Delta m_{j}=0,-1$ transitions can be neglected (for example, $\Delta / 2 \pi \sim 10$ and $\mu_{B} B / h \sim$ $100 \mathrm{MHz}$.) The emission by the induced circular dipole has a spiral wave front in the $x z$ plane, tilted with respect to the forward $\hat{\mathbf{z}}$ direction of the incident beam.

We represent the light fields by their angular spectrum [34,35], using spherical $k$-space coordinates $(k, \theta, \phi)$. For monochromatic light, with $k=\omega / c$ fixed, the incident field can be written as $\frac{1}{2} \mathbf{E}_{\text {in }}(\Omega) e^{-i \omega t}+$ c.c., with $\Omega=(\theta, \phi)$. The total field is the sum of the incident and scattered waves. Writing only the positive frequency $\left(\sim e^{-i \omega t}\right)$ components, the total field reads

$$
\mathbf{E}(\Omega)=\mathbf{E}_{\text {in }}(\Omega)+\mathbf{E}_{\mathrm{sc}}(\Omega),
$$

with $\mathbf{E}_{\mathrm{sc}}(\Omega)$ the scattered wave.

We define the radiant intensity

$$
J(\Omega)=|\mathbf{E}(\Omega)|^{2} / 2 Z_{0},
$$

with $Z_{0}=1 / \epsilon_{0} c$, so that $J(\Omega) d \Omega$ is the power flowing out of an infinitesimal solid angle $d \Omega=\sin \theta d \theta d \phi$ around

$$
\mathbf{u}_{\Omega}=(\sin \theta \cos \phi, \sin \theta \sin \phi, \cos \theta) .
$$

Combining Eqs. (1) and (2), the total radiant intensity is the sum of three terms,

$$
J(\Omega)=J_{\text {in }}(\Omega)+J_{\text {sc }}(\Omega)+J_{\text {if }}(\Omega) .
$$

The interference term

$$
J_{\mathrm{if}}(\Omega)=\frac{1}{2 Z_{0}}\left[\mathbf{E}_{\mathrm{in}}^{*}(\Omega) \cdot \mathbf{E}_{\mathrm{sc}}^{(\mathrm{coh})}(\Omega)+\text { c.c. }\right]
$$

contains only the coherent component of the scattered field. An incoherent component would contribute to $J_{\mathrm{sc}}(\Omega)$ but not to $J_{\text {if }}(\Omega)$. For simplicity we assume that the scattered field is entirely coherent, essentially restricting ourselves to the low-saturation limit (see Supplemental Material [35]).

The deflection of the light beam can be expressed as the change in average wave vector $\langle\mathbf{k}\rangle-\langle\mathbf{k}\rangle_{\text {in }}$ between the total (incident plus scattered) and the incident wave, using

$$
\langle\mathbf{k}\rangle_{\text {in }}=k \frac{\int \mathbf{u}_{\Omega} J_{\text {in }}(\Omega) d \Omega}{\int J_{\text {in }}(\Omega) d \Omega}=k \frac{\int \mathbf{u}_{\Omega} J_{\text {in }}(\Omega) d \Omega}{P_{\text {in }}}
$$

and similar for $\langle\mathbf{k}\rangle$, omitting the subscript. Assuming (again for simplicity) that nonradiative decay is absent, we shall write $P_{\text {in }}=P$ throughout.

The deflection is entirely determined by the interference term $J_{\text {if }}(\Omega)$. The scattered light itself does not contribute, due to the symmetry of the dipole radiation pattern, $J_{\mathrm{sc}}(\theta, \phi)=J_{\mathrm{sc}}(\pi-\theta, \pi+\phi)$, so that $\int \mathbf{u}_{\Omega} J_{\mathrm{sc}}(\Omega) d \Omega=0$. For the deflection, we therefore have

$$
\delta\langle\mathbf{k}\rangle=\langle\mathbf{k}\rangle-\langle\mathbf{k}\rangle_{\text {in }}=\frac{k}{P} \int \mathbf{u}_{\Omega} J_{\text {if }}(\Omega) d \Omega,
$$

and for the force on the atom, by momentum conservation,

$$
\mathbf{F}=-\frac{P}{\omega} \delta\langle\mathbf{k}\rangle=-\frac{1}{c} \int \mathbf{u}_{\Omega} J_{\text {if }}(\Omega) d \Omega .
$$

While this expression includes the forward radiation pressure force, in the cases of interest here the main force will be transverse to the optical axis, $\mathbf{F} \approx F_{x} \hat{\mathbf{x}}$. Then (approximately) $\delta\langle\mathbf{k}\rangle \perp\langle\mathbf{k}\rangle_{\text {in }}$ and with $\langle\mathbf{k}\rangle_{\text {in }} \approx k \mathbf{u}_{z}$ the deflection angle is

$$
|\delta \theta| \approx \frac{|\delta\langle\mathbf{k}\rangle|}{k} .
$$

We will choose $\delta \theta>0$ if $F_{x}<0$.

Let us now introduce specific field patterns to calculate $J_{\text {if }}(\Omega)$. We take the dipole to be circular, $\mathbf{p}=p e^{i \alpha} \mathbf{u}_{+}$, with $\mathbf{u}_{ \pm}=(\hat{\mathbf{x}} \mp i \hat{\mathbf{z}}) / \sqrt{2}$ denoting spherical unit vectors, and $\alpha$ the phase of the $p_{x}$ component of the dipole, relative to the local driving field. The field radiated by a coherent dipole [32], in angular coordinates, takes the form [35] 


$$
\mathbf{E}_{\mathrm{sc}}(\Omega)=\mathcal{E}_{\mathrm{sc}} i e^{i \alpha}\left(\mathbf{u}_{\Omega} \times \mathbf{u}_{+}\right) \times \mathbf{u}_{\Omega}
$$

with corresponding $J_{\mathrm{sc}}(\Omega)$ given by Eq. (2). Here $\mathcal{E}_{\mathrm{sc}}=$ $p k^{2} / 4 \pi \epsilon_{0}>0$ is a real-valued amplitude. Assuming the steady state of the optical Bloch equations for a twolevel system, $\cot \alpha=-\Delta / \gamma$, with $\Delta=\omega-\omega_{0}$ the detuning from the $\Delta m_{j}=+1$ transition, and $\gamma=\omega_{0}^{3} D^{2} / 6 \pi \epsilon_{0} \hbar c^{3}$ the half width of the transition, with $D$ the transition dipole moment.

For comparison, we consider two different types of incident beams, Gaussian $(G)$ and "angular top hat" $(\Pi)$, where the latter approximates the output of a uniformly illuminated focusing lens. The field for these two beams can be written as

$$
\begin{gathered}
\mathbf{E}_{\mathrm{in}}^{(G)}(\Omega) \approx \mathcal{E}_{0}^{(G)} \exp \left[-\theta^{2} / w_{\theta}^{2}\right] \mathbf{u}_{x}(\Omega), \\
\mathbf{E}_{\mathrm{in}}^{(\Pi)}(\Omega)=\mathcal{E}_{0}^{(\Pi)} \Pi\left(\theta / 2 r_{\theta}\right) \mathbf{u}_{x}(\Omega),
\end{gathered}
$$

with amplitudes $\mathcal{E}_{0}^{(G)}$ and $\mathcal{E}_{0}^{(\Pi)}>0$. The Gaussian beam has an angular width $w_{\theta}$, which is related to the minimum waist $w_{0}\left(1 / e^{2}\right.$ spatial radius of intensity $)$ as $w_{\theta} w_{0}=\lambda / \pi$. For the angular top hat, $\Pi\left(\theta / 2 r_{\theta}\right)$ is the rectangular function with angular half-width $r_{\theta}$ and unit amplitude. Its spatial profile near the focus is the familiar Airy disk pattern. Note that neither propagation phases nor the Gouy phase are visible here, as the above expressions are in angular coordinates [35].

The polarization vector $\mathbf{u}_{x}(\Omega)$ is transverse to $\mathbf{u}_{\Omega}$; it is obtained by corotating $\hat{\mathbf{x}}$ when rotating $\hat{\mathbf{z}} \rightarrow \mathbf{u}_{\Omega}$, i.e., rotating by $\theta$ around an axis $\hat{\mathbf{z}} \times \mathbf{u}_{\Omega}[11,36]$,

$$
\mathbf{u}_{x}(\Omega)=\left(\begin{array}{c}
\cos \theta \cos ^{2} \phi+\sin ^{2} \phi \\
(\cos \theta-1) \sin \phi \cos \phi \\
-\sin \theta \cos \phi
\end{array}\right) .
$$

When combining Eq. (10) with Eq. (11) or (12) in Eq. (5), the interference term contains the amplitude product $\mathcal{E}_{0}^{(G)} \mathcal{E}_{\mathrm{sc}}$ or $\mathcal{E}_{0}^{(\Pi)} \mathcal{E}_{\mathrm{sc}}$. In the low-saturation limit, the amplitude $\mathcal{E}_{\text {sc }}$ is proportional to $\mathcal{E}_{0}^{(G)}$ or $\mathcal{E}_{0}^{(\Pi)}$. Their ratio can be obtained by requiring energy conservation [35]. Upon insertion of the resulting ratios $\mathcal{E}_{\text {sc }} / \mathcal{E}_{0}^{(G)}$ and $\mathcal{E}_{\text {sc }} / \mathcal{E}_{0}^{(\Pi)}$ into Eq. (5), the interference term $J_{\text {if }}(\Omega)$ becomes proportional to the total power; the deflection angle is then independent of power.

In Fig. 2 we show $J_{\text {in }}(\Omega)$ in the plane of the dipole $(\phi=0)$, together with the total radiant intensity $J(\Omega)$. For the Gaussian beam, the effect of $J_{\text {if }}(\Omega)$ is to shift the peak and the average of the direction of propagation away from $\theta=0$. For the angular top hat, the interference leads to an intensity gradient across the angular width of the beam, whereas the edges stay at the same angle. In this case, the

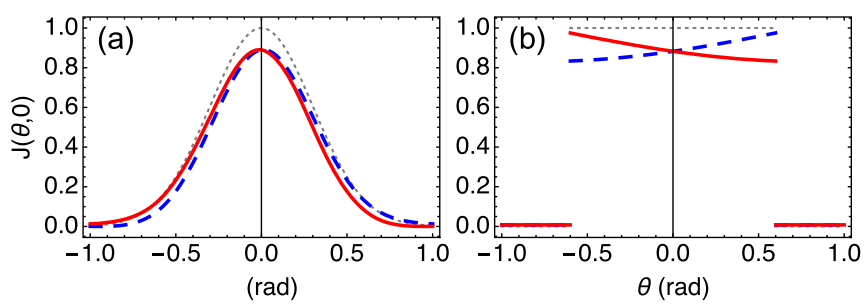

FIG. 2. Beam deflection: radiant intensities in the plane of the $\mathbf{u}_{+}$dipole, for (a) a Gaussian incident beam with $w_{\theta}=0.6$ and (b) an angular top hat incident beam with $r_{\theta}=0.6$. In both cases, the gray dotted curve shows $J_{\text {in }}(\theta, \phi=0)$ of the incident beam, normalized to 1 for $\theta=0$; red solid and blue dashed curves show the outgoing, or total $J(\theta, 0)$, for $\Delta=-\gamma$ and $+\gamma$, respectively. For clarity, we identify $(\theta, 0) \equiv(-\theta, \pi)$. Curves remain the same upon switching simultaneously the signs of the detuning and the spin of the dipole.

intensity gradient leads to a change in average beam direction.

Finally, the deflection angle is obtained by integration as in Eq. (7),

$$
\delta \theta \approx \frac{3}{4} \frac{\gamma \Delta}{\left(\gamma^{2}+\Delta^{2}\right)} \times \begin{cases}w_{\theta}^{4} & (\text { Gauss }) \\ r_{\theta}^{4} / 4 & (\text { angular top hat })\end{cases}
$$

and the reaction force as

$$
F_{x} \approx-\frac{P}{c} \delta \theta
$$

The results are given as the leading order in $w_{\theta}$ and $r_{\theta}$. The deflection angle reaches maximal values of $\delta \theta= \pm 3 w_{\theta}^{4} / 8$ and $\pm 3 r_{\theta}^{4} / 32$, respectively, for $\Delta= \pm \gamma$; it vanishes in the plane wave limit, $w_{\theta}, r_{\theta} \rightarrow 0$. In this central result, we recognize in the detuning dependence that the force is essentially a dipole force [37], arising from polarization gradients near the focus of a linearly polarized light beam [7-9,12,14,24,31].

We now address the question of how we can observe the deflection of a laser beam, either directly or via the reaction force on the atom. As shown by Eq. (14), the angle of deflection by a single atom is small compared to the divergence angle, $|\delta \theta| \ll r_{\theta}, w_{\theta}$. A direct observation will thus require sufficiently high signal-to-noise ratio, similar to what was achieved in the recent observation of apparent $\chi$ displacement of an emitter [3]. With maximal signal occurring near resonance $(\Delta= \pm \gamma)$, where the photon scattering rate is high, the best approach would be to hold the atom in an independent trap, such as an ion trap or a tight optical tweezer. One can then look for the deflection of a weak, near-resonant probe beam. A larger deflection angle may be obtained if multiple atoms cooperate. For example, one may consider dense clouds of subwavelength size, containing tens to hundreds of atoms, that have been 
observed to show collective scattering properties [38,39]. Another possibility may be to use elongated, (quasi)onedimensional samples with tight $(\lesssim X)$ radial confinement, achievable, e.g., in optical lattices [40-42] and on atom chips [43].

The second mode of observation, via the force on the atom, provides extra opportunities to manipulate spindependent atomic motion in an optical tweezer. To see this we consider case (ii): an optical tweezer trapping an atom with a $j=1 \rightarrow j^{\prime}=0$ transition. The $\left|m_{j}= \pm 1\right\rangle_{y}$ states now couple to the $\left(\sigma^{\mp}\right)_{y}$ components of the light field and therefore experience opposite forces $F_{x}$. In this configuration, there is no need for a separate probe beam [24], the far off-resonance light $(\Delta / 2 \pi \sim 1-10 \mathrm{THz})$ of the tweezer itself is sufficient. The photon scattering and associated heating rates can thus be kept as low as in typical tweezer experiments. In this case, we assume that the Zeeman shift is large compared to the trap depth $U_{0}$ (for example, $\mu_{B} B / h \sim 10$ and $U_{0} / h \sim 1 \mathrm{MHz}$.) Looking at the spiral wave of a $\mathbf{u}_{+}$dipole shown in Fig. 1, we can readily see that the relative tilt of the forward wave fronts will vanish if we displace the atom by $x$ in the $x$ direction. By thus aligning the wave fronts, the transverse force should vanish. An atom in the $\left|m_{j}=-1\right\rangle_{y}$ sublevel will therefore find an equilibrium position in the tweezer at a displaced off-axis location $x_{\mathrm{eq}}=\chi$. By the same reasoning, the $\left|m_{j}=+1\right\rangle_{y}$ sublevel will have the opposite displacement, so that for the $j=1 \rightarrow j^{\prime}=0$ transition

$$
x_{\mathrm{eq}}=-\left(m_{j}\right)_{y} \chi .
$$

The tweezer thus traps the atom off axis, where the focus is not, in a spin-dependent location. For the situation considered here, the $\left|m_{j}=0\right\rangle_{y}$ state would be untrapped, for a lack of $\pi$ component in the laser polarization. This could be changed by rotating $\mathbf{B}$. In particular, setting the angle between $\mathbf{E}_{\text {in }}$ and $\mathbf{B}$ to $\arctan (\sqrt{2})$, the polarization components $\sigma^{-}, \pi$, and $\sigma^{+}$would become equal. At this "magic angle" all three spin components would be trapped with a Stern-Gerlach type separation [24,44].

These simple geometric arguments are backed up by a calculation [35], that shows that Eq. (14) for the beam deflection is multiplied by $1 \mp k d$, for a $\mathbf{u}_{ \pm}$dipole displaced by $d$ in the $x$ direction, to lowest order in $d$. Thus, the transverse force indeed vanishes for a transverse displacement of $d=k^{-1}=\chi$ in the $x$ direction. Remarkably, the size of the displacement is independent of the detuning, the beam divergence angle, the trap frequency, or even the precise shape of the beam (Gauss vs angular top hat). This profound insight follows from the geometric properties of the scattering problem.

The off-axis trapping locations offer interesting opportunities to manipulate the motion of atoms in the tweezer, see Fig. 3. Let us imagine an atom trapped in the $\left|m_{j}=1\right\rangle_{y}$ state. As we slowly rotate the magnetic field in the $y z$ plane,

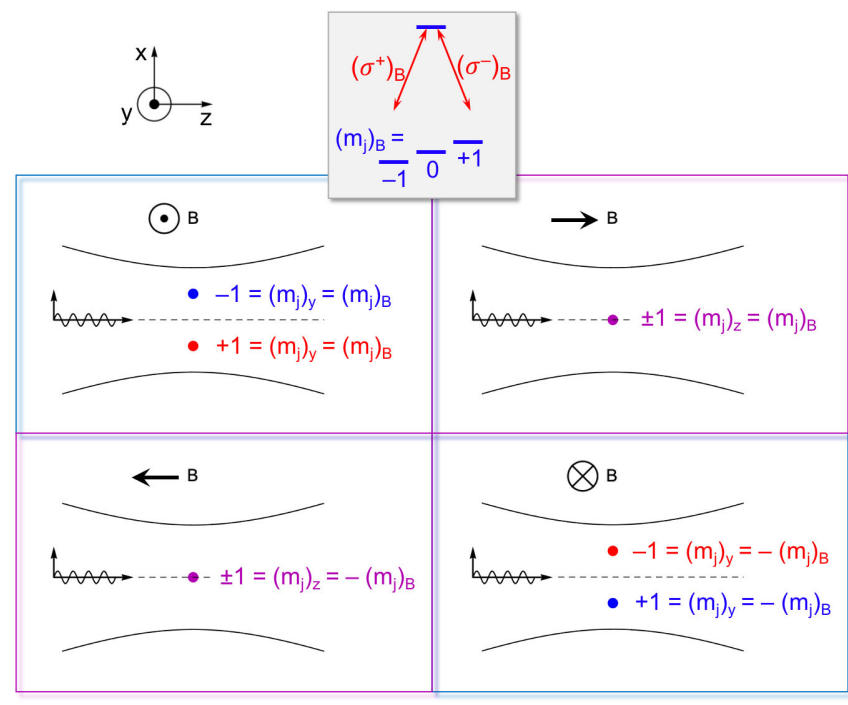

FIG. 3. Optical tweezer operating on a $j=1 \rightarrow j^{\prime}=0$ transition, leading to $\pm \chi$ off-axis displacements for the $\left(m_{j}\right)_{y}=\mp 1$ sublevels (upper left). The four panels show, in clockwise order, the effect of a rotation of the quantization axis $(\mathbf{B})$, through a cycle $y \rightarrow z \rightarrow-y \rightarrow-z$. While the $\mathbf{B}$-referenced $\left(m_{j}\right)_{B}$ of an atom is conserved, the space-referenced $\left(m_{j}\right)_{y}$ is not. The locations of the $\left(m_{j}\right)_{B}= \pm 1$ traps move up and down along the $x$ axis, in antiphase. If $\mathbf{B}$ is rotated at the trap frequency, spindependent oscillatory motion in the tweezer can be induced.

the orientation of the atom will adiabatically follow the rotating quantization axis. After rotating the field $y \rightarrow z \rightarrow-y$, the spin will have maintained its orientation relative to $\mathbf{B}$, i.e., $\left|m_{j}=1\right\rangle_{B} \rightarrow\left|m_{j}=1\right\rangle_{B}$. However, its orientation will have flipped in space, $\left|m_{j}=1\right\rangle_{y} \rightarrow$ $\left|m_{j}=-1\right\rangle_{y}$, since $\mathbf{B}$ has changed direction. The spacereferenced spin flip implies that the atom must have moved to the other side of the optical axis. Thus, by rotating the magnetic field in the $y z$ plane at a frequency $\omega_{B}$, we effectively shake the trap back and forth: $x_{\mathrm{eq}}=-\left(m_{j}\right)_{B} x \cos \omega_{B} t$. The $m_{j}= \pm 1$ levels are shaken with opposite phase.

Shaking the trap at an amplitude $\lambda$ is equivalent to a harmonic driving force $F_{x}=m \omega^{2} \chi \cos \omega_{B} t$, with $\omega$ the trap frequency. Resonant shaking, $\omega \approx \omega_{B}$, will induce an oscillatory motion in the trap. For example, for a tweezer with a laser wavelength of $\lambda \approx 0.8 \mu \mathrm{m}$, a Gaussian waist of $2 \mu \mathrm{m}$, holding an atom of mass $m=88 u$ in a $20 \mu \mathrm{K}$ deep trap, the trap frequency will be $\omega \approx 2 \pi \times 7 \mathrm{kHz}$. In a simple driven harmonic oscillator model, only 3.5 drive cycles would impart enough energy to kick the atom out of the trap, corresponding to a velocity of $\sim 6 \mathrm{~cm} / \mathrm{s}$. In reality one would of course need to take anharmonicity into account. The point here is that magnetic field modulation can easily induce oscillatory motion in the trap, which can then be detected either as trap loss, or by using time-of-flight imaging methods. For the required magnetic field, a few gauss should be sufficient to ensure that the Larmor 
frequency is large compared to the trap frequency. Rotating the field at frequencies of $\sim 10 \mathrm{kHz}$ is well possible, being comparable to what is used in time-averaged, orbiting potential traps [45].

Many available atomic-level systems should be suitable to display off-axis tweezer trapping. For example, in ${ }^{88} \mathrm{Sr}$ the transition ${ }^{3} P_{2} \rightarrow{ }^{3} S_{1}$ would provide a $j=2 \rightarrow j^{\prime}=1$ transition. The outer $\left(m_{j}\right)_{y}=2(-2)$ state couples only to the $\sigma^{-}\left(\sigma^{+}\right)$polarization component, so its spatial shift will be $-\chi(+\chi)$. Using ${ }^{87} \mathrm{Rb}$ one could operate a tweezer red detuned to the $D_{1}$ line $(795 \mathrm{~nm})$, driving the two hyperfine lines $F=2 \rightarrow F^{\prime}=1,2$. Also in this case the outer state $\left(m_{F}\right)_{y}=2(-2)$ is displaced by $-\chi(+\chi)$, as long as the detuning stays small compared to the fine structure splitting of the $D$ lines.

In summary, it is predicted that a circular dipole can deflect a focused laser beam, similar to a spinning ball deflecting a stream of air in the Magnus effect. The reaction force on the atom leads to spin-dependent, off-axis displacement of atoms trapped in an optical tweezer. For a pure circular dipole, the displacement is $\pm \chi$, independent of many trap parameters. An external magnetic field can be used to induce spin-dependent motion or to perform SternGerlach type analysis of the spin states of the atom in the tweezer.

I would like to thank N. J. van Druten, R. Gerritsma, J. Minar, and A. Urech for stimulating and encouraging discussions. This work was supported by the Netherlands Organization for Scientific Research (NWO).

r.j.c.spreeuw@uva.nl

[1] G. Magnus, Ueber die Abweichung der Geschosse, und: Ueber eine auffallende Erscheinung bei rotirenden Körpern, Ann. Phys. (Leipzig) 164, 1 (1853).

[2] C. G. Darwin, Notes on the theory of radiation, Proc. R. Soc. Ser. A 136, 36 (1932).

[3] G. Araneda, S. Walser, Y. Colombe, D. B. Higginbottom, J. Volz, R. Blatt, and A. Rauschenbeutel, Wavelength-scale errors in optical localization due to spin-orbit coupling of light, Nat. Phys. 15, 17 (2019).

[4] L. Allen, M. W. Beijersbergen, R. J. C. Spreeuw, and J. P. Woerdman, Orbital angular momentum of light and the transformation of Laguerre-Gaussian laser modes, Phys. Rev. A 45, 8185 (1992).

[5] K. Y. Bliokh and F. Nori, Transverse and longitudinal angular momenta of light, Phys. Rep. 592, 1 (2015).

[6] S. J. van Enk and G. Nienhuis, Commutation rules and eigenvalues of spin and orbital angular momentum of radiation fields, J. Mod. Opt. 41, 963 (1994).

[7] R. Dorn, S. Quabis, and G. Leuchs, The focus of lightlinear polarization breaks the rotational symmetry of the focal spot, J. Mod. Opt. 50, 1917 (2003).

[8] T. A. Nieminen, A. B. Stilgoe, N. R. Heckenberg, and H. Rubinsztein-Dunlop, Angular momentum of a strongly focused Gaussian beam, J. Opt. A 10, 115005 (2008).
[9] P. B. Monteiro, Paulo A. Maia Neto, and H. M. Nussenzveig, Angular momentum of focused beams: Beyond the paraxial approximation, Phys. Rev. A 79, 033830 (2009).

[10] K. Y. Bliokh, M. A. Alonso, E. A. Ostrovskaya, and A. Aiello, Angular momenta and spin-orbit interaction of nonparaxial light in free space, Phys. Rev. A 82, 063825 (2010).

[11] O. G. Rodríguez-Herrera, D. Lara, K. Y. Bliokh, E. A. Ostrovskaya, and C. Dainty, Optical Nanoprobing via Spin-Orbit Interaction of Light, Phys. Rev. Lett. 104, 253601 (2010).

[12] K. Y. Bliokh, E. A. Ostrovskaya, M. A. Alonso, O. G. Rodríguez-Herrera, D. Lara, and C. Dainty, Spin-to-orbital angular momentum conversion in focusing, scattering, and imaging systems, Opt. Express 19, 26132 (2011).

[13] O. V. Angelsky, A. Y. Bekshaev, P. P. Maksimyak, A. P. Maksimyak, S. G. Hanson, and C. Y. Zenkova, Orbital rotation without orbital angular momentum: Mechanical action of the spin part of the internal energy flow in light beams, Opt. Express 20, 3563 (2012).

[14] J. D. Thompson, T. G. Tiecke, A. S. Zibrov, V. Vuletić, and M. D. Lukin, Coherence and Raman Sideband Cooling of a Single Atom in an Optical Tweezer, Phys. Rev. Lett. 110, 133001 (2013).

[15] D. Barredo, S. de Léséleuc, V. Lienhard, T. Lahaye, and A. Browaeys, An atom-by-atom assembler of defect-free arbitrary two-dimensional atomic arrays, Science 354, 1021 (2016).

[16] M. Endres, H. Bernien, A. Keesling, H. Levine, E. R. Anschuetz, A. Krajenbrink, C. Senko, V. Vuletić, M. Greiner, and M.D. Lukin, Atom-by-atom assembly of defect-free one-dimensional cold atom arrays, Science 354, 1024 (2016).

[17] H. Bernien, S. Schwartz, A. Keesling, H. Levine, A. Omran, H. Pichler, S. Choi, A. S. Zibrov, M. Endres, M. Greiner, V. Vuletić, and M. D. Lukin, Probing many-body dynamics on a 51-atom quantum simulator, Nature (London) 551, 579 (2017).

[18] A. Cooper, J. P. Covey, I. S. Madjarov, S. G. Porsev, M. S. Safronova, and M. Endres, Alkaline-Earth Atoms in Optical Tweezers, Phys. Rev. X 8, 041055 (2018).

[19] M. A. Norcia, A. W. Young, and A. M. Kaufman, Microscopic Control and Detection of Ultracold Strontium in Optical-Tweezer Arrays, Phys. Rev. X 8, 041054 (2018).

[20] G. Pagano, F. Scazza, and M. Foss-Feig, Fast and scalable quantum information processing with two-electron atoms in optical tweezer arrays, Adv. Quantum Technol. 2, 1800067 (2019)

[21] N. Jackson, R. Hanley, M. Hill, F. Leroux, C. Adams, and M. Jones, Number-resolved imaging of ${ }^{88} \mathrm{Sr}$ atoms in a long working distance optical tweezer, SciPost Phys. 8, 038 (2020).

[22] L. Anderegg, L. W. Cheuk, Y. Bao, S. Burchesky, W. Ketterle, K.-K. Ni, and J. M. Doyle, An optical tweezer array of ultracold molecules, Science 365, 1156 (2019).

[23] S. Saskin, J. T. Wilson, B. Grinkemeyer, and J. D. Thompson, Narrow-Line Cooling and Imaging of Ytterbium Atoms in an Optical Tweezer Array, Phys. Rev. Lett. 122, 143002 (2019). 
[24] K.-P. Wang, J. Zhuang, X.-D. He, R.-J. Guo, C. Sheng, P. $\mathrm{Xu}$, M. Liu, J. Wang, and M.-S. Zhan, High-fidelity manipulation of the quantized motion of a single atom via Stern-Gerlach splitting, Chin. Phys. Lett. 37, 044209 (2020).

[25] I. S. Madjarov, A. Cooper, A. L. Shaw, J. P. Covey, V. Schkolnik, T. H. Yoon, J.R. Williams, and M. Endres, An Atomic-Array Optical Clock with Single-Atom Readout, Phys. Rev. X 9, 041052 (2019).

[26] M. A. Norcia, A. W. Young, W. J. Eckner, E. Oelker, J. Ye, and A. M. Kaufman, Seconds-scale coherence on an optical clock transition in a tweezer array, Science 366, 93 (2019).

[27] See also [28] for a very different kind of analogy of the Magnus effect.

[28] A. V. Dooghin, N. D. Kundikova, V. S. Liberman, and B. Y. Zel'dovich, Optical Magnus effect, Phys. Rev. A 45, 8204 (1992).

[29] C. Schwartz and A. Dogariu, Conservation of angular momentum of light in single scattering, Opt. Express 14, 8425 (2006).

[30] H. F. Arnoldus, X. Li, and J. Shu, Subwavelength displacement of the far-field image of a radiating dipole, Opt. Lett. 33, 1446 (2008).

[31] L. Caldwell and M. R. Tarbutt, Sideband cooling of molecules in optical traps, Phys. Rev. Research 2, 013251 (2020).

[32] J. D. Jackson, Classical Electrodynamics, 3rd ed. (Wiley, New York, NY, 1999), Chaps. 9 and 10.

[33] R. Schlesser and A. Weis, Light-beam deflection by cesium vapor in a transverse-magnetic field, Opt. Lett. 17, 1015 (1992).

[34] L. Mandel and E. Wolf, Optical Coherence and Quantum Optics (Cambridge University Press, Cambridge, England, 1995), Chaps. 3 and 5.

[35] See Supplemental Material at http://link.aps.org/ supplemental/10.1103/PhysRevLett.125.233201 for definitions, technical details, and supporting calculations.
[36] B. Richards and E. Wolf, Electromagnetic diffraction in optical systems, II. Structure of the image field in an aplanatic system, Proc. R. Soc. Ser. A 253, 358 (1959).

[37] J. P. Gordon and A. Ashkin, Motion of atoms in a radiation trap, Phys. Rev. A 21, 1606 (1980).

[38] J. Pellegrino, R. Bourgain, S. Jennewein, Y. R. P. Sortais, A. Browaeys, S. D. Jenkins, and J. Ruostekoski, Observation of Suppression of Light Scattering Induced by Dipole-Dipole Interactions in a Cold-Atom Ensemble, Phys. Rev. Lett. 113, 133602 (2014).

[39] S. Machluf, J. B. Naber, M. L. Soudijn, J. Ruostekoski, and R. J. C. Spreeuw, Collective suppression of optical hyperfine pumping in dense clouds of atoms in microtraps, Phys. Rev. A 100, 051801(R) (2019).

[40] H. Moritz, T. Stöferle, M. Köhl, and T. Esslinger, Exciting Collective Oscillations in a Trapped 1D Gas, Phys. Rev. Lett. 91, 250402 (2003).

[41] B. Paredes, A. Widera, V. Murg, O. Mandel, S. Fölling, I. Cirac, G. V. Shlyapnikov, T. W. Hänsch, and I. Bloch, Tonks-Girardeau gas of ultracold atoms in an optical lattice, Nature (London) 429, 277 (2004).

[42] T. Kinoshita, T. Wenger, and D. S. Weiss, Observation of a one-dimensional Tonks-Girardeau gas, Science 305, 1125 (2004).

[43] T. Jacqmin, J. Armijo, T. Berrada, K. V. Kheruntsyan, and I. Bouchoule, Sub-Poissonian Fluctuations in a 1D Bose Gas: From the Quantum Quasicondensate to the Strongly Interacting Regime, Phys. Rev. Lett. 106, 230405 (2011).

[44] S. Stellmer, R. Grimm, and F. Schreck, Sub-poissonian fluctuations in a 1D Bose gas: From the quantum quasicondensate to the strongly interacting regime, Phys. Rev. A 84, 043611 (2011).

[45] W. Petrich, M. H. Anderson, J. R. Ensher, and E. A. Cornell, Stable, Tightly Confining Magnetic Trap for Evaporative Cooling of Neutral Atoms, Phys. Rev. Lett. 74, 3352 (1995). 